\title{
LORENTZIAN GEOMETRY AND PHYSICS IN KASPAROV'S THEORY
}

\author{
KOEN VAN DEN DUNGEN
}

(Received 10 November 2015; first published online 21 January 2016)

\begin{abstract}
2010 Mathematics subject classification: primary 19K35; secondary 53C50, 58B34, 70S15. Keywords and phrases: Lorentzian geometry, gauge theory, Kasparov's theory, spectral triples.
\end{abstract}

We study two geometric themes, Lorentzian geometry and gauge theory, from the perspective of Connes' noncommutative geometry [3] and (the unbounded version of) Kasparov's $K K$-theory [2,7]. Lorentzian geometry is the mathematical framework underlying Einstein's description of gravity. The geometric formulation of a gauge theory (in terms of principal bundles) offers a classical description for the interactions between particles. The underlying motivation is the hope that this noncommutative approach may lead to a unified description of gauge theories coupled with gravity on a Lorentzian manifold.

The main objects in noncommutative geometry are spectral triples, which encompass and generalise Riemannian spin manifolds. A spectral triple defines a class in $K$-homology, via which one can access the topology of the (noncommutative) manifold. In this thesis we present two possible definitions for 'Lorentzian spectral triples', which offer noncommutative generalisations of Lorentzian manifolds as well. We will prove that both definitions preserve the link with analytic $K$-homology. We will describe under which conditions Lorentzian (or pseudo-Riemannian) manifolds satisfy these definitions. Another main example is the harmonic oscillator, which, in particular, shows that our framework allows us to deal with more than just metrics of indefinite signature.

In the context of noncommutative geometry, the description of a gauge theory can be obtained from so-called almost-commutative manifolds. While the usual approach yields by default a topologically trivial gauge theory (in the sense that the corresponding principal fibre bundle is globally trivial), we show in this thesis that the framework can be adapted, using the internal unbounded Kasparov product, to allow for globally nontrivial gauge theories as well.

Thesis submitted to the Australian National University in March 2015; degree awarded on 30 July 2015; supervisor Adam Rennie.

(c) 2016 Australian Mathematical Publishing Association Inc. 0004-9727/2016 \$16.00 
Finally, we combine the two themes of Lorentzian geometry and gauge theory, and we define Krein spectral triples, which generalise spectral triples from Hilbert spaces to Krein spaces. We use this definition to construct almost-commutative Lorentzian manifolds. Furthermore, we propose a Lorentzian alternative for the fermionic action, which allows us to derive (the fermionic part of) the Lagrangian of a gauge theory. We show that our alternative action recovers exactly the correct physical Lagrangian.

The results of this thesis are published in [1, 4-6].

\section{References}

[1] J. Boeijink and K. van den Dungen, 'On globally non-trivial almost-commutative manifolds', J. Math. Phys. 55(10) 103508 (2014).

[2] S. Baaj and P. Julg, 'Théorie bivariante de Kasparov et opérateurs non bornés dans les $C^{*}$-modules hilbertiens', C. R. Acad. Sci. Paris Ser. I Math. 296 (1983), 875-878.

[3] A. Connes, Noncommutative Geometry (Academic Press, San Diego, CA, 1994).

[4] K. van den Dungen, M. Paschke and A. Rennie, 'Pseudo-Riemannian spectral triples and the harmonic oscillator', J. Geom. Phys. 73 (2013), 37-55.

[5] K. van den Dungen and A. Rennie, 'Indefinite Kasparov modules and pseudo-Riemannian manifolds', arXiv:1503.06916 (2015).

[6] K. van den Dungen, 'Krein spectral triples and the fermionic action', arXiv:1505.01939 (2015).

[7] G. G. Kasparov, 'The operator K-functor and extensions of $C^{*}$-algebras', Izv. Akad. Nauk SSSR Ser. Mat. 44 (1980), 571-636.

KOEN VAN DEN DUNGEN, Scuola Internazionale Superiore di Studi Avanzati, SISSA, via Bonomea 265, 34136 Trieste, Italy

e-mail: kdungen@sissa.it 